\title{
Modified Bipartite Matching for Multiobjective Optimization
}

\author{
Fanglei Sun, Victor O. K. Li \\ Department of Electrical and Electronic Engineering \\ University of Hong Kong \\ Pokfulam Road, Hong Kong, China \\ Email:\{flsun, vli\}@eee.hku.hk
}

\author{
Zhifeng Diao \\ Department of Electrical Engineering \\ Arizona State University \\ AZ 85278 USA \\ Email:zhifeng.diao@asu.edu
}

\begin{abstract}
In graph theory, the Hungarian algorithm can provide the maximum weighted bipartite matching for assignment problems. In this paper, a modified bipartite matching (MBM) algorithm is proposed for multiobjective optimization. This algorithm can be widely used to solve the weighted bipartite matching problem with multiobjective optimization. We illustrate the application of MBM to antenna assignments in wireless MIMO system. The simulation results show that MBM enjoys low computational complexity and maximizes the system capacity, while keeping the fairness among mobile users.
\end{abstract}

\section{INTRODUCTION}

The Hungarian method for the assignment problem described an algorithm for constructing a maximum weight perfect matching in a bipartite graph [1]. It is proved that the Hungarian algorithm can always find the maximum assignment, i.e., an optimal solution to the maximum weight sum [1]. Compared with exhaustive search, the computational complexity is reduced to $O\left(n^{4}\right)$. However, the Hungarian method only considers single-objective optimization, limiting its application to assignments with multiobjective optimization.

In network performance analysis, a single global objective, which may be one of the network performance measures, such as throughput, is often examined to optimize network performance. This approach is meaningful in the homogeneous situation, such as when each user has the same quality of service (QoS) requirement. In this case, equal performance is the implied fairness criterion, and global optimality is equivalent to individual optimization. However, in the heterogeneous case, individual requirements may be ignored by using a single overall objective, and sometimes users may have to sacrifice their own performances for the good of the entire network [2] [3].

In wireless MIMO communication systems based on spatial multiplexing, a data stream is split into multiple parallel substreams, and each substream is transmitted through one of the transmit antennas [4]. Combined with multiuser diversity [5] in cellular packet transmission systems, a couple of antenna assignment schemes have been proposed for packet schedulers to maximize the system capacity or to provide fairness among

This research is supported in part by the Research Grants Council of the Hong Kong Special Administrative Region, China, under Grant No. HKU $7148 / 06 \mathrm{E}$. mobile users [6]-[8]. However these scheduling schemes only consider single-objective optimization. None of them consider multiobjective optimization which account for both capacity maximization and fairness.

In this paper, a modified bipartite matching (MBM) algorithm is proposed for multiobjective optimization, such as maximizing the total throughput, while keeping the fairness among individual users. In MBM, this is realized by applying the Hungarian algorithm to each updated bipartite graph and choosing the matching with the largest fairness index among the updated matchings. The bipartite graph is updated by reducing one edge at a time till no edges remain in the graph. The deleted edge is the one violates the fairness criterion most seriously. For each deletion, after the processing of the Hungarian algorithm, the maximum weighted matching is also guaranteed for the updated bipartite graph. Consequently, the fairness comparison can be finished in $O\left(n^{2}\right)$ and the matching which has the best fairness performance is the final solution. In this paper, MBM is applied to a MIMO communication system as an example. Simulation results show that it can effectively guarantee the system capacity and fairness performance. More importantly, our proposed MBM algorithm can be widely applied to job assignments and resource allocation problems which need multiobjective optimization.

The paper is organized as follows. Section II presents the details of the MBM algorithm. In Section III, we give an example of MBM for antenna assignments in wireless MIMO communication systems. Section VI draws the conclusions of this paper.

\section{Description OF MBM}

\section{A. Multiobjective Optimization Functions}

A graph is denoted by $G(V, E)$, where $V$ is the vertex set and $E$ is the edge set of the graph. If $V=V_{1} \cup V_{2}$ with $V_{1} \cap V_{2}=\Phi$, and each edge in $E$ has one endpoint in $V_{1}$ and the other in $V_{2}$, the graph $G(V, E)$ is a bipartite graph, which can also be denoted as $G\left(V_{1}, V_{2}, E\right)$. The bipartite graph is very useful for some applications, such as an assignment problem which can be depicted as follows: Given a weighted complete bipartite graph $G=(X \cup Y, X \times Y)$, where edge $(x, y)$ has the weight $w(x, y)$, find a matching $M$ from $X$ to $Y$ with maximum weight. In an application, $X$ could be a 
set of workers, $y$ could be a set of jobs, and $w(x, y)$ be the profit made by assigning worker $x$ to job $y$. The essence of the assignment problem is to find the optimal matching. Basically, there are two approaches to the optimization problem: singleobjective overall weight optimization and multiobjective individual optimization.

For the overall weight optimization, we have a singleobjective optimization problem:

$$
\max \sum_{x \subset X, y \subset Y} w(x, y), \quad(x, y) \subset E .
$$

This approach is often used to obtain the optimal performance for the entire assignment, and the meaning of optimization is straightforward. To consider the individual performance, we resort to the multiobjective approach. For example, the system is required to keep profit fairness among the workers. Consider any matching $M_{j}(j=1, \ldots n)$, where $n$ is the maximum number of matchings chosen from $G$. We assume the objective evaluation function is $f\left(w_{j}(x, y)\right)$, where $(x, y) \subset M_{j}$ and $w_{j}(x, y)$ represents the weights of edges in $M_{j}$. Consequently, the multiobjective optimization problem is to combine (1) with

$$
\begin{aligned}
& \max f\left(w_{j}(x, y)\right), \quad(x, y) \subset M_{j}, \\
& j=1, \ldots, n \text {. }
\end{aligned}
$$

The multiobjective functions can be constructed based on specific applications, and are not limited to examples we have given in this paper. The main contribution of our work is to introduce a modified bipartite matching algorithm to provide a solution to general multiojective optimization problems.

\section{B. MBM Algorithm}

Since individual objectives may conflict with each other, and may conflict with the entire optimization objective, a new modified bipartite matching algorithm is proposed. It has been proved that the Hungarian algorithm always find the maximum weighted matching for a bipartite graph. That means it can provide an optimal solution to the above problem (1). The following is the description of the procedure of the Hungarian algorithm. Matrix $\mathbf{W}=\left[w_{i j}\right]$ has elements $w_{i j}$, which is the weight of assigning worker $i$ to job $j$ as shown in Fig. 1 (a).

1) Step 1: Let $X, Y$ be the bipartite sets. Initialize two labels $u_{i}$ and $v_{j}$ by $u_{i}=\max _{j} w_{i j}, v_{j}=0, i, j=1, \ldots, k$. In Fig. 1 (b), the numbers written at the left and the top of the matrix express the values of $u_{i}$ and $v_{j}$, respectively.

2) Step 2: Obtain the excess matrix $\mathbf{C}$ by the following. $c_{i j}=u_{i}+v_{j}-w_{i j}$.

3) Step 3: Find the subgraph $G$ that includes vertices $i$ and $j$ satisfying $c_{i j}=0$ and the corresponding edge $e_{i j}$. Then find the maximum matching $M$ in $G$ and underline the entries in $M$. If $M$ is a perfect matching with $k$ edges, the optimal assignment is obtained.

4) Step 4: Let $Q$ be a vertex cover of $G$, and let $R=X \cup Q$ and $T=Y \cap Q$. The vertex cover $Q$ as a vertex set of $G$ which contains at least one endpoint of each edge. Now find $\epsilon$ satisfying $\epsilon=\min \left\{c_{i j}: x_{i} \in X-R, y_{j} \in Y-T\right\}$. For
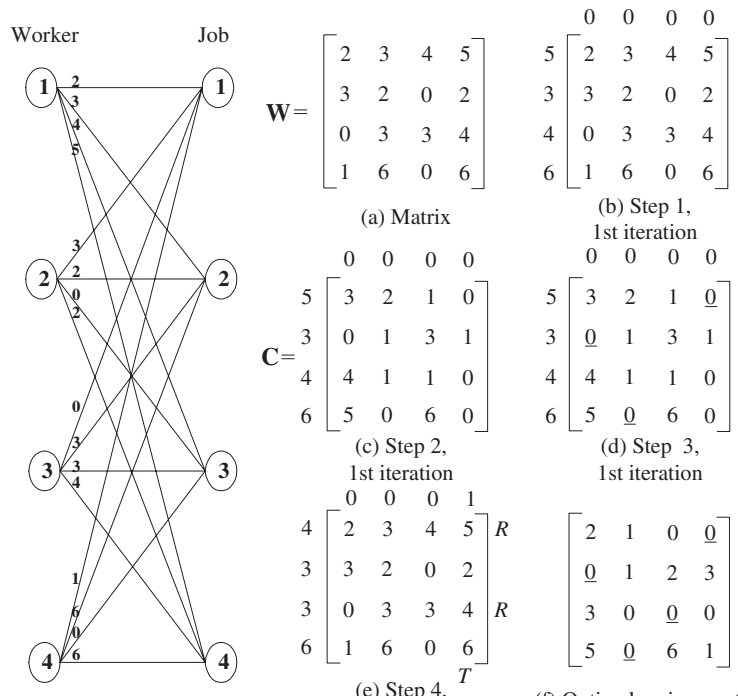

(a) Matrix

$\begin{array}{llll}0 & 0 & 0 & 0\end{array}$

$\mathbf{C}={ }_{4}^{3}\left[\begin{array}{lll}3 & 2 & 1 \\ 0 & 1 & 3 \\ 4 & 1 & 1\end{array}\right.$

1st iteration

$\begin{array}{llll}0 & 0 & 0 & 0\end{array}$

$5\left[\begin{array}{llll}3 & 2 & 1 & \underline{0}\end{array}\right]$

$3 \underline{0} \quad \begin{array}{llll}0 & 1 & 3 & 1\end{array}$

$\begin{array}{lllll}4 & 4 & 1 & 1 & 0\end{array}$

$6 \quad \begin{array}{llll}6 & \underline{0} & 6 & 0\end{array}$

(d) Step 3,

1st iteration

(c) Step 2

1st iteration

$\begin{array}{llll}0 & 0 & 0 & 1\end{array}$

$4\left[\begin{array}{llll}2 & 3 & 4 & 5\end{array}\right] R$

$\begin{array}{lllll}3 & 3 & 2 & 0 & 2\end{array}$

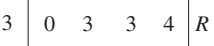

$6\left[\begin{array}{llll}1 & 6 & 0 & 6\end{array}\right]$

(e) Step 4, 1st iteration

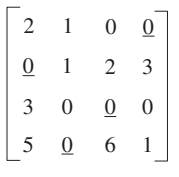

(f) Optimal assignment

Fig. 1. Weighted bipartite matching.

example, if $\epsilon$ equals 1 in Fig. 1, decrease $u_{i}$ by $\epsilon$ for the rows of $R$ and increase $v_{j}$ by $\epsilon$ for the columns of $T$. Then go to step 2.

Steps 2 to 4 are repeated until the perfect matching $M$, namely the optimal assignment, is obtained.

After the procedure of the Hungarian algorithm, the maximum weighted matching $M$ can be achieved, and for simplicity, we denote the weight of the $k$ edges as $w_{i}, i=1, \ldots, k$. To consider the multiobjective fairness optimization problem (2), MBM calculates $\eta_{i}$ of the different weighted edges by

$$
\eta_{i}=\left|w_{i}-\left(\sum_{i=1}^{k} w_{i}\right) / k\right|^{2}, \quad i=1, \ldots, k .
$$

In terms of the fairness, these $\eta_{i}$ values represent the distance from the mean. Then we chose the edges $m$ by

$$
\{m\}=\operatorname{argmax}_{i}\left\{\sigma_{i}\right\}, \quad i=1, \ldots, k .
$$

For the original maximum matching, denoted as $M_{1}$, we can deduce that the edge $m$ violates the fairness criterion most seriously. So in order to improve the fairness performance, MBM deletes the edge $m$ in $G$, namely, changes the corresponding element to zero in the matrix $\mathbf{W}$. With the new updated weight matrix, we apply the Hungarian algorithm again to find the new maximum matching, and then delete the next edge in the new case. MBM will iterate these operations till there is no edge in $G$. If we only consider the square matrix W with $N \times N$ elements, the iterations can be finished in at most $O\left(n^{2}\right)$ times. Given each obtained maximum matching $M_{j}(j=1, \ldots, N \times N)$, their corresponding fairness can be evaluated by the defined evaluation function $f\left(w_{j}(x, y)\right)$. Then the final solution given by MBM is 


$$
\begin{aligned}
& \{p\}=\operatorname{argmax}_{j}\left\{f\left(w_{j}(x, y)\right)\right\}, \\
& \quad(x, y) \subset M_{j}, j=1, \ldots, N \times N .
\end{aligned}
$$

For multiobjective optimization, based on the Hungarian algorithm, $M_{j}$ is guaranteed to be the maximum weighted matching for each updated bipartite graph. With the deleting operation of the edge with the most serious fairness violation in the previous matching, the result of the fairness function is updated. By comparing the fairness performances in $O\left(n^{2}\right)$ calculations, the final solution $M_{p}$ can achieve the best fairness among those maximum weighed matchings for each updated $G$. Therefore, MBM can effectively solve the multiobjective optimization problems.

\section{EXAMPLE}

In this section, MBM is applied for antenna assignments in the downlink of an MIMO communication system to maximize the overall channel capacity, while keeping the fairness among all mobile users.

\section{A. System Model}

The system model is shown in Fig. 2, where the base station has $N_{T}$ antennas and each mobile user has $N_{R}\left(\geq N_{T}\right)$ antennas. The number of mobile users is $K$. The transmit power is equally divided among the transmit antennas. The receiver estimates the post-detection signal-to-noise ratio (SNR) of each transmit antenna, and passes the SNR information to the base station through the uplink feedback channel at the start of each time-slot (of duration Ts). Based on this information, the scheduler selects a group of users and assigns the antennas to them to transmit in the time-slot. The channel encounters path loss and shadow fading, and it is time-invariant during each time-slot and independent between time-slots. Also, the channel is assumed to be spatially uncorrelated. The channel matrix $H_{k}(t)$ between the base station and user $k$ at time-slot $t$ is [5]

$$
H_{k}(t)=\sqrt{S N R_{0} \cdot\left(l_{k} / L\right)^{-\beta} \cdot 10^{S_{k} / 10}} \cdot G_{k}(t),
$$

where $S N R_{0}$ is the median $S N R, L$ is the cell radius, $G_{k}(t)$ is an independent complex Gaussian random variable with zero mean and unit variance, $\beta$ is an path loss exponent, and $S_{k}(t)$ is a real Gaussian random variable with zero mean and variance of $\sigma_{S}^{2}$. In addition, $l_{k}$ is the distance between the base station and user $k$. The distance of each user from the base station is randomly initialized between zero and $L$. The postdetection SNR [5] is defined as the SNR of a transmit symbol after minimum mean-squared error (MMSE) detection, and the corresponding value for the transmit antenna $n$ to user $k$ can be calculated as

$\gamma_{k, n}(t)=\frac{\left|\left[W_{k}(t) H_{k}(t)\right]_{n n}\right|^{2}}{\left(\sigma_{N}^{2} / P_{R}\right) N_{T} \sum_{m=1}^{N_{T}}\left|\left[W_{k}(t)\right]_{n m}\right|^{2}+\sum_{m=1, m \neq n}^{N_{T}}\left|\left[W_{k}(t) H_{k}(t)\right]_{n m}\right|^{2}}$,

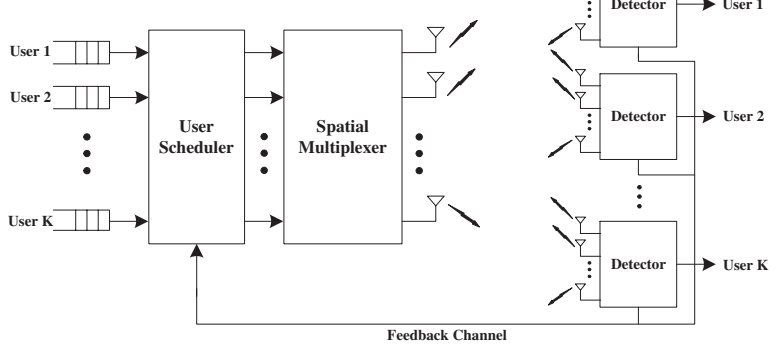

Fig. 2. Downlink MIMO system.

where $P_{R}$ is the total received signal power and $\sigma_{N}^{2}$ is the noise power per receive antenna. The weight matrix for MMSE is given as

$$
W_{k}(t)=H_{k}^{H}(t)\left(H_{k}(t) H_{k}^{H}(t)+\left(\sigma_{N}^{2} N_{T} / P_{R}\right) I_{N_{R}}\right)^{-1},
$$

where $(\cdot)^{H}$ denotes the conjugate transpose and $I_{N_{R}}$ is the $N_{R} \times N_{R}$ identity matrix. Therefore, the system capacity for time-slot $t$ can be expressed as

$$
C(t)=\sum_{n=1}^{N_{T}} \log _{2}\left(1+\gamma_{k, n}(t)\right)
$$

\section{B. Problem Formulation}

In spatial multiplexing, a data stream is split into multiple substreams, and each substream is transmitted through a transmit antenna. To exploit multiuser diversity, the scheduler can simultaneously choose as many multiple users as transmit antennas and allocate an antenna to each user. As the capacity is a function of SNR, maximizing the sum of the weight obtained from SNR is equivalent to the capacity maximization of (9). We further denote $C\left(k_{1}, \ldots, k_{N_{T}}\right)$ as the capacity achieved by allocating transmit antenna $i$ to user $k_{i}$ for $i=$ $1, \ldots, N_{T}$. Consequently, our proposed MBM aims to search $k_{1}, \ldots, k_{N_{T}}$, for $\forall i, j(\neq i) ; k_{i} \neq k_{j}$ to

$$
\max C\left(k_{1}, \ldots, k_{N_{T}}\right)=\max \sum_{i=1}^{N_{T}} \log _{2}\left(1+\gamma_{k_{i}, i}\right) .
$$

In wireless communication system, the fairness index is widely used to evaluate the fairness performance [9]. It takes values between 0 and 1 , and is defined as follows:

$$
f=\frac{\left(\sum_{i=1}^{N_{T}} C_{k_{i}, i}\right)^{2}}{N_{T}\left(\sum_{i=1}^{N_{T}} C_{k_{i}, i}^{2}\right)}
$$

where $C_{k_{i}, i}=\log _{2}\left(1+\gamma_{k_{i}, i}\right)$ is the transmit rate achieved by assigning antenna $i$ to user $k_{i}$. We assume each user has the same QoS requirement on throughput. As presented in Section II-B, after each update of the graph $G$ by deleting the edge chosen by (4), a new maximum weight matching is 
TABLE I

COMPUTATIONAL COMPLEXITIES

\begin{tabular}{|c|c|}
\hline Assignment scheme & Computational complexity \\
\hline \hline Hungarian Algorithm & $O\left(n^{4}\right)$ \\
\hline Pure fairness & $O(n !)$ \\
\hline MBM & $O\left(n^{6}\right)$ \\
\hline
\end{tabular}

obtained till there is no edge in $G$. For each achieved matching $M_{j}$, the corresponding fairness index $f_{j}\left(j=1, \ldots, N_{T} \times\right.$ $N_{T}$ ) could be calculated by (11). Therefore the multiobjective optimization is achieved by choosing the maximum $f_{p}$ among the updated matchings, namely,

$$
\{p\}=\operatorname{argmax}_{j}\left\{f_{j}\right\}, j=1, \ldots, N_{T} \times N_{T} .
$$

Finally, the maximum matching $M_{p}$ should be the solution for the multiobjective capacity and fairness optimization problem.

\section{Simulation Results}

In this paper, we consider a $4 \times 4$ MIMO system, and evaluate the capacity and fairness performance for the besteffort traffics. We assume $S N R_{0}=10 \mathrm{~dB}$, the path loss exponent $\beta=3.7 \mathrm{~dB}$, the $\log$ standard deviation of shadow fading $\sigma_{S}=8 \mathrm{~dB}$, the cell radius $L=1 \mathrm{Km}$, and $P_{R} / \sigma_{N}^{2}=$ $10 \mathrm{~dB}$. In each time slot, if $K<N_{T}$, we assume some users can be assigned multiple antennas by a round Robin (RR) scheduler or other schemes. If $K>N_{T}$, an asymmetric bipartite matching is constructed. In this case, we append $\left(K-N_{T}\right)$ all-zero rows to the weighted matrix, then deal with the problem as a symmetric bipartite matching. Our main goal is to test the performance of MBM for antenna assignments, so for simplicity, we set $K=N_{T}$. In this model, the distance of each user from the base station is uniformly distributed between 0 and $L$. Simulation time is $2 \times 10^{3}$ Ts. We compared the performances of the Hungarian maximum weighted matching, the pure fairness maximum matching, and our proposed MBM algorithm. Table I compares the computational complexities of these three schemes. For MBM, given a certain fairness index requirement, $O\left(n^{6}\right)$ is the upper bound of the search complexity.

Fig. 3 shows the throughput performance comparisons. For the generated network scenario in our simulation, the Hungarian algorithm can provide the maximum system capacity of around $33.65 \mathrm{bps} / \mathrm{Hz}$ and a fairness index of around 0.77 . If we assume the system fairness optimization target is to have a fairness index above 0.8 , the Hungarian algorithm is inadequate. MBM can optimize the throughput for any given fairness requirements. We show the MBM throughput for fairness requirements $(f)$ from 0.8 to 0.9 . By exhaustive search, the pure fairness maximization scheme gives the best fairness performance of over 0.9 , but it sacrifices the system capacity.

\section{CONCLUSions}

In this paper, based on the Hungarian algorithm, a modified bipartite matching (MBM) algorithm is proposed for multi-

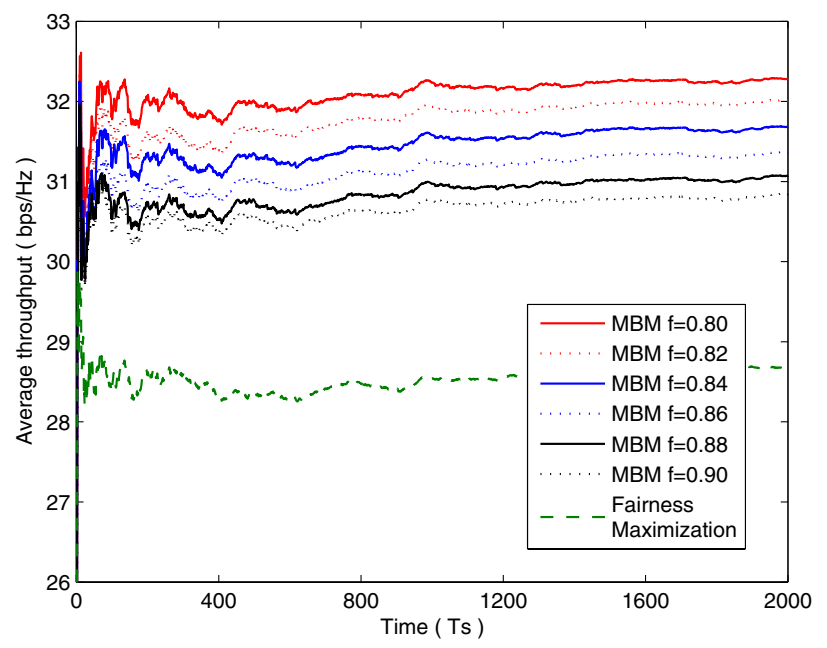

Fig. 3. Throughput comparisons.

objective optimization. This algorithm can effectively balance conflicting objectives in multiobjective optimization problems, and is widely applicable to resource assignment problems. In this paper, we illustrate the application of MBM to antenna assignments in wireless MIMO system. The simulation results show that MBM enjoys low computational complexity and maximizes the system capacity, while keeping the fairness among mobile users.

\section{REFERENCES}

[1] H. W. Kuhn, "The Hungarian Method for the Assignment Problem," Naval Research Logistic Quarterly, vol. 2, pp. 83-97, 1955.

[2] K. J. Runser, J. M. Gorce, and S. Ubeda, "QoS Constrained Wireless LAN Optimization Within a Multiobjective Framework," IEEE Wireless Communcations, vol. 13, no. 6, pp. 26-33, 2006.

[3] B. Wu and Q. Wang, "A Multiobjective Analytic Framework for Slotted ALOHA Wireless LANs, " IEEE ICUPC, pp. 12-16, Tokyo, Japan, Nov. 1995.

[4] P. W. Wolniansky, C. J. Foschini, G. D. Golden, and R. A. Valenzuela, "V-BLAST: An architecture for Realizing High Data Rates over the Rich-scattering Wireless Channel," URSI Int Symp. Signals, Systems. ond Electronics, pp. 295-300, Pisa, Italy, Sept.-Oct. 1998.

[5] R. W. Heath, M. Airy, and A. I. Paulraj, "Multiuser Diversity for MIMO Wireless Systems with Linear Receivers," Asilomor Conf. Signals, Systems, and Computers, pp. 1194-1199, Pacific Grove, CA, USA, Nov. 2001.

[6] O. S. Shin and K. B. Lee, " Antenna-assisted Round Robin Scheduling for MIMO Cellular System," IEEE Communication Letters, vol. 7, no. 3, pp. 109-111, Mar. 2003.

[7] Y. J. Choi, J. Kim, and S. Bahk, ’Downlink Scheduling with Fairness and Optimal Antenna Assignment for MIMO Cellular Systems," IEEE GLOBECOM, vol. 5, pp. 3165-3169, Dallas, Texas, USA, Nov. 2004.

[8] M. Torabzadeh and Y. Ji, "Efficient Assignment of Transmit Antennas for Wireless Communications," IEEE International Conference in Central Asia, pp. 1-5, Tashkent, Uzbekistan, Sept. 2006.

[9] R. Jain, D. Chiu, and W. Hawe, "A Quantitative Measure of Fairness and Discrimination for Resource Allocation in Shared Computer Systems," DEC Research, Tec. Report, TR-301, 1984. 